\title{
OFICINAS DE FORMAÇÃO DE PROFISIONAIS DA EQUIPE SAÚDE DA FAMÍLIA PARA A GESTÃO DO ACOLHIMENTO COM CLASSIFICAÇÃO DE RISCO
}

\author{
TRAINING WORKSHOP FOR PROFESSIONALS FAMILY HEALTH \\ TEAM FOR THE MANAGEMENT OF THE RECEPTION WITH \\ CLASSIFICATION OF RISK
}

\section{TALLER DE FORMACIÓN PARA LOS PROFESIONALES DEL EQUIPO DE SALUD FAMILIAR PARA LA GESTIÓN DE LA RECEPCIÓN DE CLASIFICACIÓN DE RIESGO}

\author{
Heloisa de Carvalho Torres ${ }^{*}$ \\ Renata Batista Lelis ${ }^{* *}$
}

\begin{abstract}
RESUMO
Descreve-se a experiência da capacitação das Equipes de Saúde da Família de uma unidade básica de saúde em relação à classificação de risco no acolhimento. A metodologia utilizada para a realização da capacitação foi a de oficinas de formação em saúde, com o objetivo de discutir o acolhimento com classificação de risco, reconhecer as necessidades do grupo e proporcionar o atendimento ao usuário de forma ágil e efetiva. Os resultados obtidos com essas oficinas possibilitaram um aprendizado significativo em relação a conteúdos teóricos de semiologia e primeiros socorros importantes para o exame e atendimento adequados ao usuário durante o acolhimento. Além disso, os participantes mostraram-se satisfeitos com o aprendizado. As oficinas revelaram-se uma estratégia importante para a capacitação dos profissionais de saúde em relação à classificação de risco no acolhimento.
\end{abstract}

Palavras chave: Capacitação, equipes de saúde, classificação de risco.

\begin{abstract}
This paper describes the training experience of a Family Health Staff from a primary care unit in classifying risk at admission. Training was carried out at health workshops, which aimed to discuss risk classification at admission as well as recognize team needs and provide effective care. Results gathered from these workshops provided significant learning of theory related to semiology and first aid, which are important instruments for a good examination and care at admission. Besides, participants were pleased with the outcome. Health workshops showed to be an important training strategy for health professionals regarding risk classification at admission.
\end{abstract}

Key words: Training, health staff, risk classification.

\footnotetext{
* Enfermeira, professor adjunto da Escola de Enfermagem da Universidade Federal de Minas Gerais. Brasil. E-mail: heloisa@enf.ufmg.br.

** Aluna do Curso de Graduação da Escola de Enfermagem da Universidade Federal de Minas Gerais. Brasil. E-mail: rblelis@ig.com.br
} 


\section{RESUMEN}

Describe la experiencia de capacitación de los Equipos de Salud de la Familia de una unidad básica de salud en cuanto a la clasificación de riesgo en la acogida. La metodología empleada para realizar la capacitación fue talleres de salud con el objetivo de discutir la acogida con clasificación de riesgo, reconocer las necesidades del grupo y proporcionar atención ágil y efectiva al usuario. Los resultados indican aprendizaje significativo sobre contenidos teóricos de semiología y primeros auxilios, importantes para examinar y atender adecuadamente al usuario en la acogida. Además, los participantes se sintieron satisfechos con el aprendizaje. Los talleres se revelaron como una importante estrategia para capacitar profesionales de salud en cuanto a la clasificación de riesgo en la acogida.

Palabras clave: Capacitación, equipos de salud, clasificación de riesgo.

Fecha recepción: 16/02/09 Fecha aceptación: 30/04/10

\section{INTRODUÇÃO}

No processo de trabalho do Programa Saúde da Família (PSF), a porta de entrada para o serviço é o acolhimento que é um modo de operar os atendimentos em saúde a todos os usuários da comunidade e uma das ferramentas utilizadas para acolher o indivíduo é a escuta de forma qualificada (1-3). Cabe aos profissionais de saúde assumir uma postura capaz de acolher, escutar e pactuar respostas mais adequadas aos usuários com resolutividade e responsabilização, orientando, quando for o caso, o indivíduo e a família em relação a outros serviços de saúde para a continuidade da assistência e buscando estabelecer uma articulação entre esse serviço visando garantir a eficácia desses encaminhamentos (4-7).

De forma, a tornar o acolhimento de forma ágil e efetiva surge a idéia de implantação da avaliação da Classificação de Risco no Acolhimento que consiste em um processo dinâmico de identificação dos indivíduos que necessitam de tratamento imediato, de acordo com o potencial de risco, agravos à saúde ou grau de sofrimento (8-10). A avaliação com Classificação de Risco busca agilidade no atendimento a partir da análise, sob a óptica de protocolo pré-estabelecido, do grau de necessidade do usuário, proporcionando atenção centrada no nível de complexidade e não na ordem de chegada $(4,10)$.
A execução da Classificação de Risco a avaliação primária deve basear-se no protocolo de situação de queixa, e tal avaliação pode se dar por explicitação dos usuários ou pela observação de quem acolhe, sendo os casos encaminhados para a classificação de risco pelo enfermeiro. Além disso, acolhimento com classificação de risco deve ser dinâmico, contínuo e incluir atividades que tranqüilizem o usuário e seus familiares, trazendo-lhes apoio emocional e segurança. Informações claras sobre o tempo de espera e destino de cada paciente, onde o mais grave é priorizado em relação ao menos grave, geram confiança no sistema $(1,4,10)$.

A implantação da classificação de risco no acolhimento assume relevância ao poder gerar vários benefícios para o atendimento, como a diminuição da ansiedade dos profissionais e usuários, melhoria das relações interpessoais na equipe de saúde, padronização de dados para estudos, pesquisas e planejamentos, e aumento da satisfação do usuário, uma vez que este será atendido de forma mais rápida e efetiva, mudando o foco da doença para o doente em uma abordagem integral do indivíduo $(4,10)$. Permite ainda problematizar sobre o que precisa ser transformado para a melhoria da qualidade da assistência, representando o esforço nobre de converter a rede pública de saúde em um espaço de ensino-aprendizagem no exercício do trabalho. Surge, desta forma, a necessidade de que os profissionais envolvidos 
em tal processo estejam preparados para este tipo de atendimento, e tal objetivo pode ser alcançado pela capacitação das equipes de saúde, realizada pelos enfermeiros, por meio de estratégias educativas (9).

O Ministério da Saúde propôs a capacitação dos profissionais como política de transformação das práticas de formação, de atenção, de gestão, de participação popular e de controle social no setor da saúde. Essa educação se baseia na aprendizagem significativa, que pode ser entendida como aprendizagem associada ao trabalho, ou seja, ela acontece no cotidiano dos profissionais e das organizações $(5,6,9)$. Com base em Freire $(4,5)$, acreditamos que as ações educativas realizadas na perspectiva dialogal, reflexiva e crítica poderá ser efetiva como instrumento para a formação de uma consciência crítica e, assim, possibilitará compreender a realidade do usuário visando prestar um atendimento com qualidade.

Desse modo, a proposta de trabalhar a educação dos profissionais de saúde da família- ESF desenvolvendo ações educativas sobre classificação de risco e envolvendo a participação de profissionais de serviço, de docente e aluno mostrou-se apropriada para gerar uma reflexão dos profissionais de saúde da família, em relação o "fazer" para o "pensar como está sendo feito", abrindo oportunidades para o diálogo entre os diversos saberes, assim como a construção de conhecimento e formação do pensamento crítico e emancipatório dos profissionais das ESF envolvidos no atendimento aos usuários.

Nesse contexto, a Universidade Federal de Minas Gerais - UFMG, em parceria com a Unidade Básica de Saúde busca envolver docentes e estudantes na educação dos profissionais, por meio da disciplina Estágio Supervisionado em Enfermagem I. Observouse a necessidade de inserção da classificação de risco no acolhimento como uma forma de tornar o atendimento aos usuários um processo mais eficiente, que priorizasse casos mais graves e ao mesmo tempo reduzisse o tempo de espera pelo atendimento. Além disso, está em andamento a implantação do protocolo de classificação de risco em hospitais de Belo Horizonte, sendo que tal protocolo deverá ser seguido pelas unidades básicas de saúde em um futuro próximo. Portanto, percebeu-se a necessidade de sensibilizar e capacitar os profissionais das equipes de saúde da família para a futura implantação do Acolhimento com Classificação de Risco nesta unidade de saúde.

Em vista do exposto, o objetivo do presente estudo é apresentar o delineamento das oficinas de formação em saúde sobre a classificação de risco no acolhimento para as Equipes de Saúde da Família de uma unidade básica de saúde da cidade de Belo Horizonte/ MG além de, reconhecer as necessidades do grupo e proporcionar o atendimento ao usuário de forma ágil e efetiva.

\section{METODOLOGÍA}

A disciplina Estágio Supervisionado em Enfermagem I, ofertada no $8^{0}$ período do curso de graduação em enfermagem da Universidade Federal de Minas Gerais - EE/UFMG, desenvolve um projeto para atualizar e qualificar as Equipes de Saúde da Família-ESF em uma Unidade Básica de Saúde-UBS desde 2007, em parceria com outras universidades e com o próprio serviço.

A técnica metodológica do trabalho em oficinas constitui-se na formação de espaços de promoção da aprendizagem que inclui a participação coletiva, multiprofissionalidade e interdisciplinaridade mediante o diálogo, contribuindo para a construção de novos conhecimentos, fortalecendo os objetivos da educação em saúde (11). O estudo foi realizado no período de agosto a dezembro de 2008, utilizando a técnicas de oficinas educativas sobre Acolhimento com Classificação de Risco.

Os pressupostos deste trabalho têm suas 
bases na concepção crítica do campo da educação em saúde e na teoria freiriana (5) que propõe a educação dialógica como forma de resgatar o conhecimento e a experiência advindas na prática social. Essa perspectiva emancipatória de educação guarda coerência com a necessidade de buscar um fundamento dialético -que articula teoria e prática- para analisar os conhecimentos sobre o Acolhimento com Classificação de Risco dos profissionais.

Inicialmente, foi realizada uma reunião com a gerente da unidade onde foi discutida a necessidade de capacitação da equipe de saúde da família em relação à classificação de risco, para que estes selecionassem as prioridades de atendimento dos usuários no acolhimento. Todos os profissionais foram convidados com antecedência, por meio de cartazes afixados em locais visíveis nas dependências da unidade. Logo em seguida, realizou-se uma reunião com a equipe, envolvendo a participação dos profissionais, para apresentação e discussão da proposta de trabalho, solicitando-os que manifestassem suas opiniões a respeito do assunto e sugestões de temas a fazerem parte da capacitação.

Participaram das oficinas de formação em saúde 17 profissionais da unidade de saúde inseridos nas quatro equipes de saúde da família e que concordaram e aceitaram em integrar este estudo, sendo nove auxiliares de enfermagem, quatro enfermeiras, uma médica, uma agente comunitária de saúde (ACS), e uma técnica em higiene dental (THD). Observa-se que são pessoas com diferentes níveis de formação, com distintas funções e atribuições, comprometidas com um produto final que é definido pelo serviço. Há, portanto, grande diversidade de papéis profissionais e ampla heterogeneidade. As agendas dos profissionais da equipe saúde da família foram reservadas para o dia e horário previsto de cada encontro.

As oficinas educativas sobre o Acolhimento com Classificação de Risco tiveram como objetivo educar e atualizar os profissionais e foram organizadas em sete encontros, sendo trabalhados assuntos e utilizadas estratégias facilitadoras da aprendizagem em cada encontro. As oficinas foram realizadas no salão da UBS, onde ocorreram quinzenalmente com duração de uma hora. Os integrantes foram posicionados em círculo a fim de facilitar a discussão dos assuntos abordados. As oficinas foram coordenadas pela enfermeira/ serviço, docente e discente de enfermagem em estágio no centro de saúde em questão.

Os conteúdos programáticos abordados foram: exame físico geral; sinais vitais, ausculta pulmonar e cardíaca; feridas e curativos; classificação de risco; dispnéia; tipos de dor e sinais de emergência. Os temas foram abordados de forma lúdica e interativa por jogos educativos e estudos de casos, para que cada um dos presentes pudesse aprender e ao mesmo tempo externar seus conhecimentos, compartilhando-os com os demais membros da equipe.

Durante as oficinas foram registrados em um diário de campo os comportamentos dos participantes, a receptividade das atividades propostas e a relação entre a equipe.

No final foi aplicado um instrumento de avaliação das oficinas com os 17 profissionais de saúde centrada em cinco questões: metodologia, conteúdo, tempo de duração, material didático e aproveitamento das oficinas.

O projeto foi aprovado pelo Comitê de Ética em Pesquisa (No parecer 90/2008) e todos os profissionais de saúde da família assinaram o Termo de Livre Consentimento.

\section{RESULTADOS E DISCUSSÃO}

Observamos no inicio do processo que o maior resultado obtido foi à aproximação entre a universidade e o serviço. Essa articulação entre o ensino e serviço constitui instância privilegiada para conduzir ações conjuntas e experiências inovadoras, de forma a contribuir para a implementação de 
um sistema de atenção à saúde de qualidade, eqüitativo e inclusivo.

Foram distribuídos e devolvidos dezessete questionários entre todos os participantes das oficinas e todos foram respondidos. A distribuição de acordo com a categorização dos profissionais se deu da seguinte maneira: auxiliares de enfermagem 53\%, enfermeiras $23 \%$, médicos $6 \%$, ACS $6 \%$, THD $6 \%$ e recepcionista $6 \%$. Em relação ao conteúdo abordado nas oficinas ser satisfatório ou não, $100 \%$ dos participantes responderam que sim, o conteúdo foi satisfatório.

Quanto ao tempo programado para a realização das oficinas, $94,1 \%$ dos participantes responderam que foi satisfatório e 5,9\% responderam que não, deveria haver mais tempo dedicado às oficinas. Cem por cento dos participantes considerou adequado o material didático utilizado nas oficinas.

No primeiro encontro para realização das oficinas sobre "Acolhimento com Classificação de Risco" apresentou-se a proposta de trabalho, a qual foi muito bem recebida pelos profissionais. Vários comentaram sobre a falta de capacitação da equipe e de como é importante tal oportunidade. Todos opinaram sobre o que deveria ser discutido nas reuniões e foi feito um cronograma das oficinas. O preparo dos profissionais para atuarem na Equipe de Saúde da Família (ESF) deve ser acompanhado, uma vez que ainda não há consenso sobre as necessidades específicas de qualificação neste cenário $(5,12)$.

Por outro lado, percebeu-se a dificuldade de adesão das auxiliares de enfermagem na primeira e segunda oficinas. Estudos comprovam a existência de carências significativas no grupo de auxiliares e técnicos de enfermagem, em relação a atualizações e capacitações. Isto se deve certamente a lacunas na formação, formação tardia e não atualização após a entrada no mercado de trabalho, fato possível de reversão se houver treinamentos adequados $(12,13)$.

$\mathrm{Na}$ terceira oficina, sobre feridas e curativos, algumas auxiliares de enfermagem re- lataram só saber a prática de curativos e não possuírem nenhum embasamento teórico. Surgiram muitas dúvidas sobre qual tipo de cobertura a ser usada para cada tipo de ferida. Todos gostaram do encontro, fizeram perguntas e compararam a teoria com a prática. Alguns autores $(6,9,14)$ afirmam que a aprendizagem acontece a todo o momento em que nos questionamos sobre algum problema atual e procuramos a resposta, a partir de uma lógica de necessidade de aprendizagem. O primeiro passo para organizar essa questão de educação é procurar perceber quais as necessidades de aperfeiçoamento relativo ao nosso cotidiano, dentro da realidade do nível de atenção à saúde na qual estamos inseridos.

Durante a quarta oficina, sobre a classificação de risco, foi informado aos profissionais que o protocolo de acolhimento com classificação de risco deverá ser implantado em todos os centros de saúde no próximo ano. Os participantes mostraram-se muito preocupados com a possibilidade do surgimento de sobrecarga de trabalho com a implantação do protocolo de classificação de risco nos centros de saúde. Autores $(11,15)$ consideram que o caráter hierárquico, burocrático e especializado das organizações de saúde somado à falta de recursos humanos e materiais, levam à sobrecarga de trabalho dos profissionais de saúde, os quais exercerem diversas funções ao mesmo tempo, em breve espaço de tempo e com poucos recursos.

A quinta, sexta e sétima oficinas agradaram aos participantes no sentido de serem uma revisão de conteúdos importantes para a prática (dispnéia, tipos de dor e sinais de emergência) e muitos casos foram exemplificados pela rotina vivenciada na unidade básica. A necessidade de adaptar os conhecimentos adquiridos à realidade vivenciada na profissão é relatada em alguns estudos $(9,10$, 14, 16). A capacitação permite aos participantes resgatar sua própria prática educativa e também estar mais preparado para atender e acolher o mesmo processo no outro. 
Assim, a oficina caracterizou-se como um processo rico de trocas de experiência entre o serviço e a academia, numa via de mão dupla. O processo de atualização e as estratégias adotadas para tal, foram avaliados positivamente pela maioria dos participantes. Relataram que a capacitação teórico-prática possibilita a implantação do protocolo do acolhimento. Os participantes manifestaram o desejo de dar continuidade às oficinas como forma de atualização e avaliação permanente da educação da equipe multidisciplinar no atendimento ao usuário.

As limitações do estudo foram relacionadas a falta de tempo dos participantes, divididos entre as atividades do centro de saúde e a realização das oficinas. Alguns problemas identificados foram relativos a organização e planejamento das gerentes do serviço em não conseguir direcionar as atividades dos profissionais para outros horários, dificultou a participação da equipe em todas as oficinas. As auxiliares de enfermagem depararam-se com um acréscimo em suas atividades com a decisão de que os ACSs não ajudariam mais na retirada de prontuários para o acolhimento e consultas médicas. Além disso, percebeu-se o medo da sobrecarga de trabalho com o surgimento de um novo protocolo de atendimento.

Deve-se destacar ainda que o centro de saúde em questão estava passando por uma mudança de gestão no período de realização das oficinas, ou seja, a gerente aposentou-se e no momento estava sendo feito um revezamento entre as enfermeiras na gerência. Tal situação gerou grande estresse entre os profissionais.

\section{CONCLUSÃO}

De qualquer forma, a despeito das limitações apresentadas durante a realização das oficinas, a grande maioria dos participantes mostrou-se satisfeita com conteúdo abor- dado, tempo programado, material didático utilizado e aproveitamento individual das oficinas, inclusive mostrando-se interessados na possibilidade de continuação de tal projeto no próximo ano. Com certeza tal disposição resultará na continuidade da capacitação e conseqüentemente, os profissionais estarão preparados para a implantação do protocolo de classificação de risco na rotina do acolhimento. As oficinas geraram discussões construtivas sobre os temas abordados.

A atualização dos profissionais de saúde permitiu-lhes que refletissem sobre o acolhimento com classificação de risco na unidade básica de saúde, em relação ao atendimento ao usuário. Pretende-se que a educação dos profissionais proporcionem a identificação de problemas, cuja solução deve ser procurada por eles próprios, mediante a busca de conhecimentos e mudança de atitudes. O processo de atualização dos profissionais de saúde deva ser contínuo dentro dos serviços e que as universidades participem ativamente desse processo.

Dessa forma, a proposta de trabalhar com os profissionais de saúde da atenção primária na modalidade de oficinas de formação em saúde sobre o acolhimento com classificação de risco para a qualidade do atendimento ao usuário envolvendo a participação dos profissionais de ensino (docentes), alunos e serviço mostrou-se apropriada para gerar uma reflexão da equipe de saúde sobre a realidade vivenciada pelos usuários e do acolhimento.

A proposta de oficina como modalidade educativa favoreceu a discussão dos desafios e dificuldades para a estruturação da prática do acolhimento com classificação de risco.

\section{REFERÊNCIAS}

1. Albino RM, Grosseman S, Riggenbach V. Classificação de risco: uma necessidade inadiável em um serviço de emergência de qualidade. ACM arq. catarin. med. 
2007; $36(4): 70-75$.

2. Brasil, Ministério da Saúde. Guia Prático do PSF; 2001.

3. Brasil, Ministério da Saúde. SecretariaExecutiva. Núcleo Técnico da Política Nacional de Humanização. Humaniza SUS: acolhimento com avaliação e classificação de risco: um paradigma éticoestético no fazer em saúde /Ministério da Saúde, Secretaria-Executiva, Núcleo Técnico da Política Nacional de Humanização, Brasília: Ministério da Saúde; 2004.

4. Ministério da Saúde, Brasil. Política Nacional de Educação Permanente. Portaria No 198/GM/MS. Em 13 de fevereiro de 2004B. Hallado em: http://www.ead. ufms/ambiente/programas/saude_educacao/arminda/portariagm198polos.pdf [Acceso em 25/10/2006].

5. Rocha Chagas N, Costa Ramos I, da Silva LF, Macedo Monteiro AR, de Melo Fialho A V. Cuidado crítico y creativo: contribuciones de la educación de Paulo Freire para la enfermería Cienc. Enferm. 2009; 15(2): 35-40.

6. Brasil, Ministério da saúde. Manual de regulação Médica das Urgências; 2006.

7. Brasil, Ministério da saúde. Boas práticas de humanização na atenção e na gestão do Sistema Único de Saúde: acolhimento com classificação de risco na emergência do Hospital Nossa Senhora da Conceição em Porto Alegre. Ministério da Saúde; 2006.

8. Ceccim RB. Educação permanente em saúde: desafio ambicioso e necessário. Interface-comunicação, saúde, educação (Botucatu). 2005; 9(16):161-168.

9. Ferreira CSW. Acolhimento com classifi- cação de risco: experiência na urgência. PNH/MS; 2006.

10. Ferretti CJ. Formação profissional e reforma do ensino técnico no Brasil: anos 90. Educ. Soc. 1997; 18 (59). Hallado em: http://www.scielo.br/scielo.php [Acesso em 25/10/2006].

11. Torres HC, Aburachid DFF, Soares SM. Oficinas de educação em saúde: uma estratégia no controle do diabetes mellitus tipo II no programa de saúde da família em Belo Horizonte/Brasil. Diabetes Clínica. 2007; 11(2):177-181.

12. Ribeiro ECO, Motta JIJ. Educação Permanente como estratégia na reorganização dos serviços de saúde. Universidade Federal da Bahia - Instituto de Saúde Coletiva - Secretaria executiva da Rede IDA, Brasil. Hallado em: http://www. redeunida.org.br/arquivos/educacao.rtf [Acesso em 25/10/2006].

13. Santos JM, Oliveira EB, Moreira AC. Estresse, fator de risco para a saúde do enfermeiro em Centro de Terapia Intensiva. Rev Enferm UERJ. 2006;14(4):580-5.

14. L’Abbate S. Comunicação e educação: uma prática de saúde. En: Merhy EL, Onocko R. Agir em saúde: um desafio para o público. São Paulo: Hucitec; 1997. Pp. 267-92.

15. Neto FRGX, Costa MCF, Rocha J, Cunha ICKO. Auxiliares e técnicos de enfermagem na saúde da família: perfil sociodemográfico e necessidades de qualificação. Trab. Educ. Saúde. 2008; 6(1): 51-64.

16. Marziale MHP, Rozestraten RIA. Turnos alternantes: fadiga mental de enfermagem. Rev. Latino-Am. Enfermagem.1995;3(1):59-78. 